IMECE2009-10872

\title{
PROBABILISTIC APPROACH TO CONDITIONAL PROBABILITY OF RELEASE OF HAZARDOUS MATERIALS FROM RAILROAD TANK CARS DURING ACCIDENTS
}

\author{
D. Y. Jeong \\ Volpe National Transportation Systems Center \\ US Department of Transportation \\ Cambridge, Massachusetts, USA
}

\begin{abstract}
This paper describes a probabilistic approach to estimate the conditional probability of release of hazardous materials from railroad tank cars during train accidents. Monte Carlo methods are used in developing a probabilistic model to simulate head impacts. The model is based on the physics of impact in conjunction with assumptions regarding the probability distribution functions of the various factors that affect the loss of lading. These factors include impact velocity, indenter size, tank material, tank diameter, effective collision mass, and tank thickness. Moreover, each factor is treated as a random variable characterized by its assumed distribution function, mean value, and standard deviation (or variance). Reverse engineering is performed to back-calculate the mean values and standard deviations of these random variables that reproduce trends observed in available accident data. The calibrated model is then used to conduct a probabilistic sensitivity analysis to examine the relative effect of these factors on the conditional probability of release. Results from the probabilistic sensitivity analysis indicate that the most significant factors that affect conditional probability of release are impact velocity, effective collision mass, and indenter size.
\end{abstract}

\section{INTRODUCTION}

Conditional probability is the probability that an event will occur given that another event has already occurred. In the present context, conditional probability of release is the probability that release of hazardous material from a railroad tank car will occur given that an accident (e.g., a train derailment or a car-to-car collision) has already occurred.

Estimates for conditional probability of release have been calculated by applying logistic regression analysis [1] to accident data contained in the Tank Car Accident Damage Database, which is maintained by the Railway Supply Institute and the Association of American Railroads (AAR). These data have been collected since the 1960s. ${ }^{1}$ Here logistic refers to the functional form of the mathematical equations used to characterize the influence of different factors on the probability of lading loss during an accident. Regression means that the mathematical equations were curve-fit to represent the data. Lading loss is assumed to occur from four specific sources: (1) releases from head impacts, (2) releases from shell impacts, (3) releases from top fittings, and (4) releases from bottom fittings. The regression assumes that the most significant factors contributing to the probability of lading loss from each source are: (1) whether a head shield is present and if so what type, (2) thickness of the tank head, (3) whether the tank is jacketed or not, (4) whether double-shelf couplers are present, (5) thickness of the tank shell, (6) whether the car is pressurized or not, and (7) if the accident occurred in a yard or on mainline track. The results of the regression analysis and the trends for conditional probability of release based on the analysis are described in detail in reference [1].

A probabilistic approach to examine accidental releases of hazardous materials from tank cars is reasonable since no two accidents are identical. For example, factors such as load severity and material properties tend to vary from one accident to the next. Moreover, probabilistic analysis can account for randomness and uncertainty in these factors.

A probabilistic model to predict the percentage of accidents that result in brittle fracture and lading loss was developed in the 1990s [2, 3]. The model was based on probabilistic fracture mechanics analysis in which a probability distribution was assumed for cracks or defects that may exist in the tank structure (presumably near welds). Failure by brittle fracture was assumed when the stress intensity factor for a given combination of accident loading and defect size exceeded the fracture toughness of the tank car

\footnotetext{
${ }^{1}$ The database contains over 40,000 records of tank cars damaged in accidents [1].
} 
steel. Subsequently the model was used to assess the potential safety benefit from constructing tank cars with steels exhibiting higher strength and toughness properties [4].

A probabilistic model is described in this paper that estimates conditional probability of release from head impacts without the assumption of pre-existing defects or cracks in the tank structure. Failure is defined in the model as puncture of the commodity tank. Moreover, accident loading and puncture resistance of the commodity tank are expressed in terms of energy quantities. That is, puncture is assumed to occur when the kinetic energy associated with a given impact scenario exceeds the energy to puncture (i.e., resistance) for a given tank car configuration or design. These energy quantities consist of different factors that are treated as random variables in the probabilistic model. Mean values and standard deviations of the random variables are backcalculated by matching the simulation results to those from the logistic regression of the accident data. After the probabilistic model is calibrated in this manner, a probabilistic sensitivity analysis is performed to examine the relative effect of the various factors on conditional probability of release.

\section{PROBABILISTIC MODEL}

Figure 1 illustrates the fundamental concept in applying the probabilistic approach to estimate probability of failure [5]. The figures shows two variables; one relating to the demand on the system, $S$ (e.g., load on the structure), and the other to the resistance of the structure, $R$. Both $S$ and $R$ are considered as random variables that are characterized by their mean values $\mu_{\mathrm{S}}$ and $\mu_{\mathrm{R}}$, standard deviations $\sigma_{\mathrm{S}}$ and $\sigma_{\mathrm{R}}$, and probability density functions $f_{S}$ and $f_{R}$. Moreover, the overlap in the two probability density functions defines the probability of failure. If the probability functions are known and can be expressed mathematically, the probability of failure can be calculated from the following integral

$$
p_{f}=\int_{\infty} f_{s}(s) F_{R}(s) d s
$$

where $F_{R}$ is the cumulative probability function representing the resistance of the structure. In general, however, exact mathematical expressions for the probability density functions are unknown. In this paper, Monte Carlo methods are used to evaluate the integral in equation (1) to estimate the probability of failure.

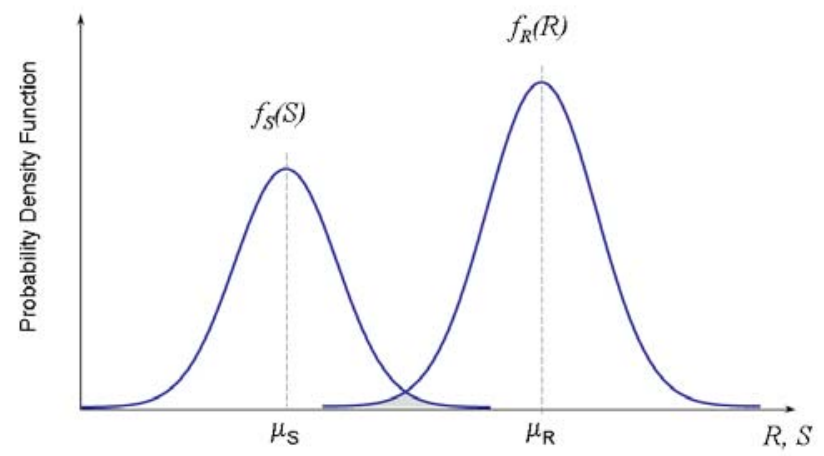

Figure 1. Schematic of Probabilistic Approach

In the probabilistic model described in this paper, the demand on the tank car during an accident is defined as the kinetic energy associated with the impact while the resistance to puncture is defined in terms of energy to puncture. Kinetic energy, $E_{K}$ consists of two parts: (1) effective collision mass, $m_{e}$ and (2) impact velocity, $v_{i}$, and is expressed mathematically as

$E_{K}=\frac{1}{2} m_{e} v_{i}^{2}$

Puncture resistance in terms of energy to cause puncture is assumed to comprise four parts: (1) indenter size in terms of the perimeter of the contacting surface $P$, (2) tank material in terms of the ultimate tensile strength of the tank car steel $\sigma_{U}$, (3) tank head thickness $h$, and (4) tank diameter $D$. The functional form of energy to puncture is assumed as a powerlaw relation:

$$
E_{P}=C P^{a} \sigma_{U} h^{b} D^{c}
$$

where $C, a, b$, and $c$ are constants to be determined. For dimensional consistency,

$$
a+b+c=3
$$

The functional form of equation (3) for puncture resistance is similar to that assumed in semi-empirical analysis of puncture during excavation of pipelines [6].

Moreover, the factors listed in equations (2) and (3) are treated in the model as random variables with assumed probability distributions as listed in Table 1. Appendix A lists the mathematical expressions for the different probability distributions assumed in the model. 
Table 1. Random Variables and Assumed Distributions

\begin{tabular}{|l|l|}
\hline Random Variable & Assumed Distribution \\
\hline Effective collision mass & Weibull \\
\hline Impact velocity & Weibull \\
\hline Indenter size & Normal \\
\hline Tank material & Log Normal \\
\hline Tank thickness & \\
\hline Tank diameter & Triangular or Uniform \\
\hline
\end{tabular}

Figure 2 shows a schematic of the probabilistic model. Monte Carlo methods are used to estimate the conditional probability of release. That is, the distribution of accident loading is calculated using equation (2) while the distribution for the puncture resistance of the tank structure is calculated using equation (3).

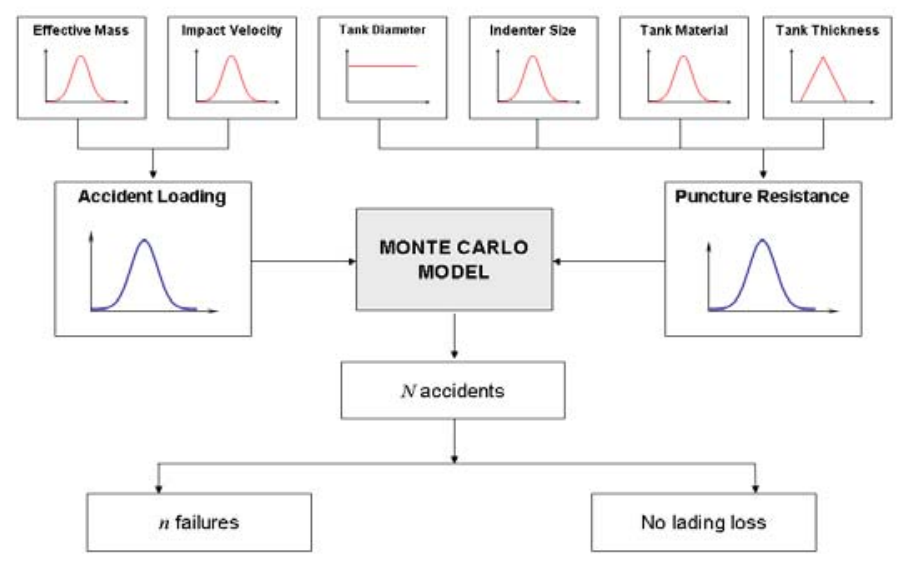

Figure 2. Schematic of Simulation Model

\section{MODEL APPLICATION}

The probabilistic model is applied to produce two different sets of results. In the first, tank thickness is treated as a deterministic variable with values ranging between 7/16 and 1 inch. In these cases, the model was exercised to simulate 100,000 accidents per thickness value. Mean values and standard deviations of the various random variables were varied iteratively to reproduce trends observed in the available accident data for conditional probability of release as a function of thickness. In the second set of results, tank thickness is treated as a random variable with an assumed probability distribution, and the calibrated model is then used to conduct a probabilistic sensitivity analysis to examine the relative effect of each factor (or random variable) on the conditional probability of release.

\footnotetext{
2 Tank thickness is a deterministic variable in the model calibration, and is treated as a random variable in the probabilistic sensitivity analysis.
}

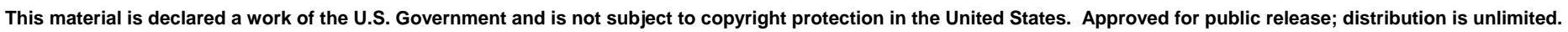

Table 2. Mean and Standard Deviation for Random Variables

\begin{tabular}{|c|c|c|}
\hline Random Variable & Mean, $\mu$ & $S D, \sigma$ \\
\hline Effective mass, lb-s ${ }^{2} / \mathrm{ft}$ & 7000 & 2800 \\
\hline Impact velocity, mph & 30 & 10 \\
\hline Indenter perimeter, inches & 60 & 9 \\
\hline Ultimate tensile strength, ksi & 88.8 & 6.7 \\
\hline Tank diameter, inches ${ }^{\mp}$ & 105 & 75 \\
\hline
\end{tabular}

\section{Calibration with Accident Data}

Figure 3 shows the trend of conditional probability of release as a function of tank head thickness for head impacts involving tanks without head shields and without jackets. The open symbols represent results from the logistic regression analysis of accident data [1]. The dashed lines represent the 95 percent confidence band on the mean value determined by the regression analysis. The solid symbols represent results from the Monte Carlo model based on the assumed probability distributions listed in Table 1. Table 2 lists the mean values and standard deviations for the random variables assumed in the model to produce these results. The values for the mean and standard deviation for the ultimate tensile strength are based on tensile strength measurements conducted on TC128B tank car steel by Southwest Research Institute [7]. The other values listed in the table are calculated from applying inverse or reverse engineering to match the regression results. In addition, the constants in equation (3); namely, $C, a, b$, and $c$; are calculated from reverse engineering to be equal to 74 , $1.5,0.5$, and 1.0 respectively. The relationships between the mean values and standard deviations of the random variables and the parameters that characterize the different probability functions are given in Appendix A. The results from the Monte Carlo simulation show a slightly stronger effect of thickness on conditional probability of release than the regression on the accident data. Moreover, the simulation results are within the 95 percent confidence band for the available accident data over the range of thicknesses.

Figure 4 compares the model results with the regression results on the accident data for head losses from jacketed tank cars without head shields. The jacket thickness of 0.119 inch is added to the tank head thickness to produce the model results. In this case, the model overestimates the conditional probability of release compared to the accident data for almost the entire range of thicknesses. The model results are approximately equal to the upper-bound confidence bound at thicknesses slightly less than 1 inch, but generally overestimate the accident data trend. Similarly, Figure 5 compares results from the model with accident data for tank cars with head shields and without jackets. As in the jacketed

\footnotetext{
‡ Mean and standard deviation are based on uniform probability density function assuming a minimum tank diameter of 90 inches and a maximum of
} 
case, the head shield thickness of 0.5 inch is added to the head thickness to produce the model results. In this case, the simple addition of the shield and tank head thicknesses produces results that are within the 95 percent confidence bands on observed in available data.

\section{Probabilistic Sensitivity Analysis}

Probabilistic sensitivity analysis is performed using the calibrated model for tank heads without head shields and without jackets to estimate the relative effect of the various factors assumed to affect conditional probability of release. Three different measures of probabilistic sensitivity are calculated in the present study: (1) probabilistic importance factor, (2) probabilistic sensitivity with respect to changes in the mean value $\mu$, and (3) probabilistic sensitivity with respect to changes in the standard deviation $\sigma$. The interpretation of these sensitivity measures and the specific details of the probabilistic sensitivity analysis are described in Appendix B.

In the probabilistic sensitivity analysis, tank thickness is treated as a random variable. Moreover, two distribution functions are assumed for tank thickness in the sensitivity analysis, triangular and uniform. Table 3 lists the mean values and standard deviations for the different distribution functions, which are based on assuming a minimum thickness of 7/16 inch, a maximum of 1 inch, and a mode of 25/32 inch. Moreover, these values are calculated using equations listed in Appendix A for these assumed distribution functions.

\section{Table 3. Mean Values and Standard Deviations for Tank Thickness and Different Distribution Functions}

\begin{tabular}{|l|c|c|}
\hline Assumed Distribution & Mean, $\mu$ & $S D, \sigma$ \\
\hline Triangular & 0.771 & 0.127 \\
\hline Uniform & 0.719 & 0.026 \\
\hline
\end{tabular}

Figure 6 shows the probabilistic importance factors for the six random variables assumed in the Monte Carlo model to estimate the conditional probability of release. Results are shown for the two different assumptions regarding the probability distribution for thickness. Similarly, Figure 7 shows the probabilistic sensitivities with respect to changes in the mean of the random variables. Figure 8 shows the probabilistic sensitivities with respect to changes in standard deviation. The length of the bars in these figures indicates the relative effect of the various random variables on the conditional probability of release. For example, each measure of probabilistic sensitivity indicates that impact velocity has the most significant effect on the estimate of conditional probability of release.

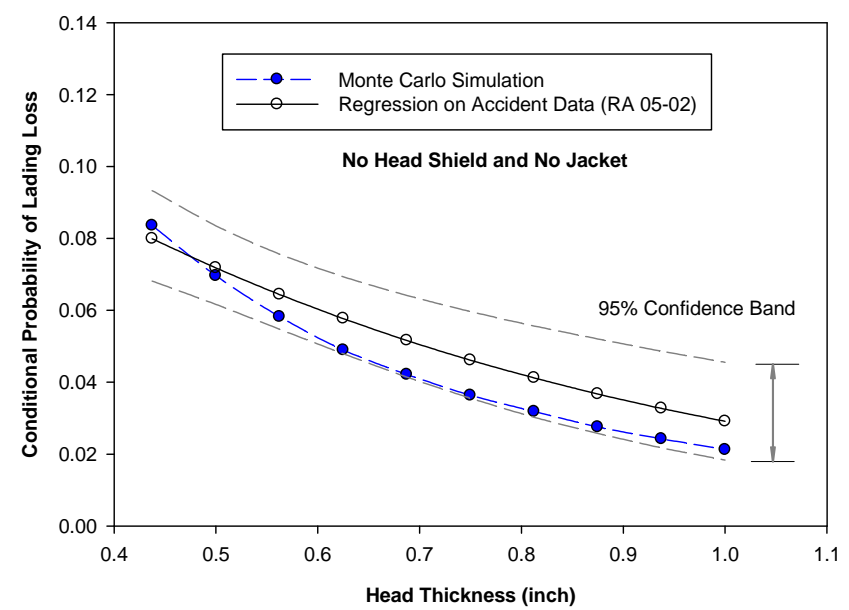

Figure 3. Conditional Probability of Release for Head Losses (No Head Shield and No Jacket)

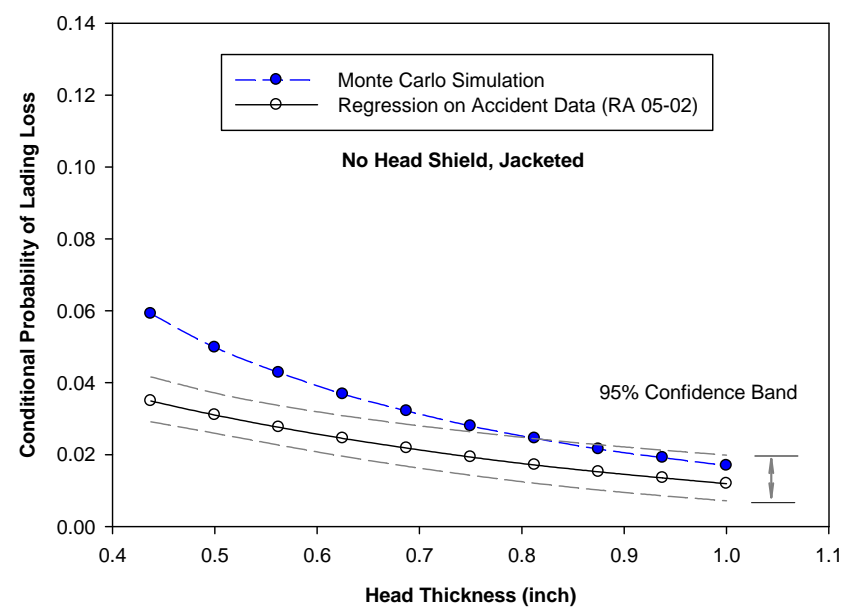

Figure 4. Conditional Probability of Release for Head Losses (No Head Shield, Jacketed)

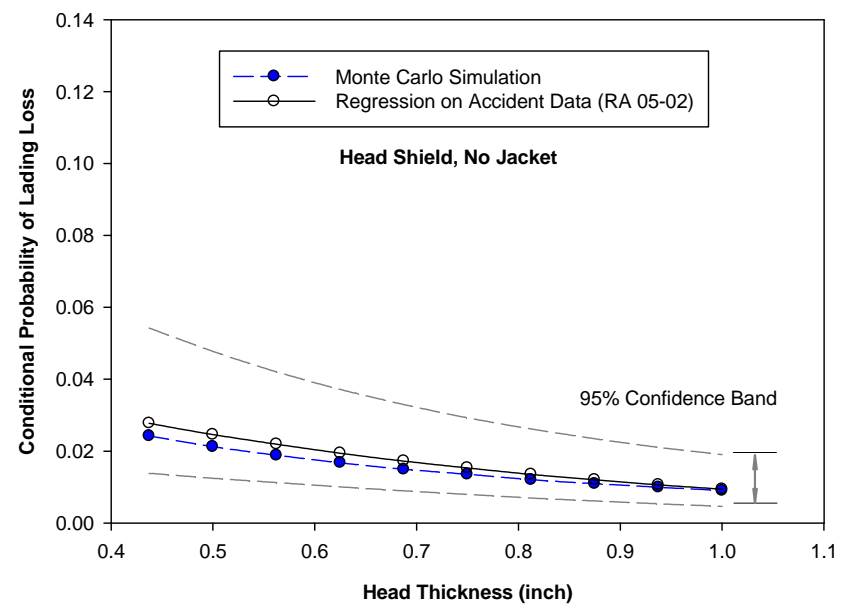

Figure 5. Conditional Probability of Release for Head Losses (Head Shield, No Jacket) 


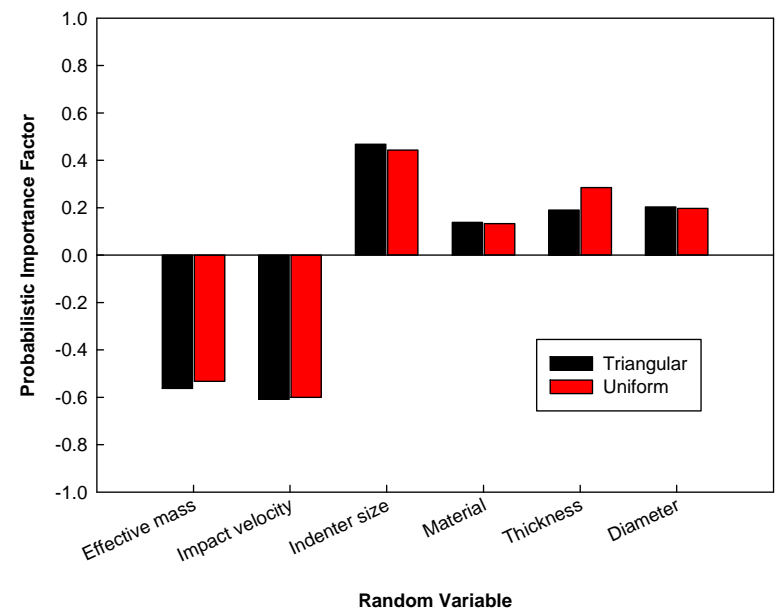

Figure 6. Probabilistic Importance Factors

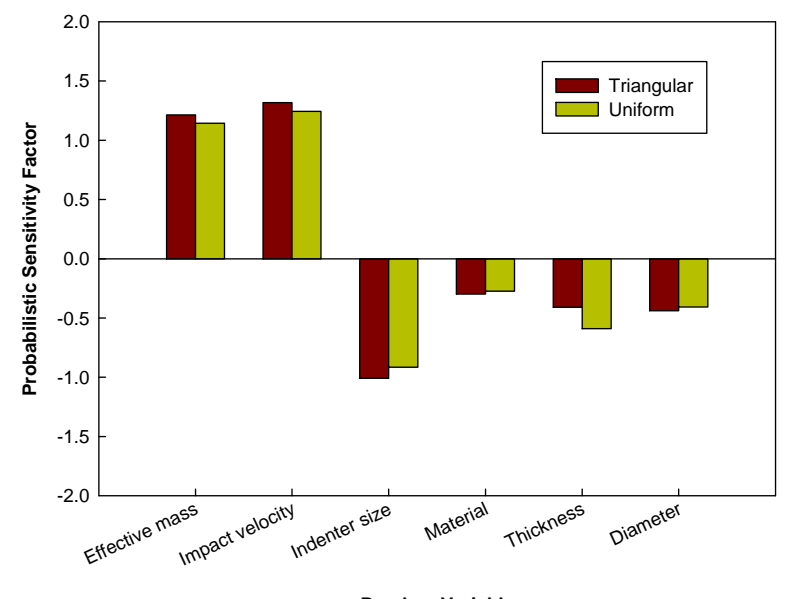

Figure 7. Probabilistic Sensitivity with Respect to Changes in Mean

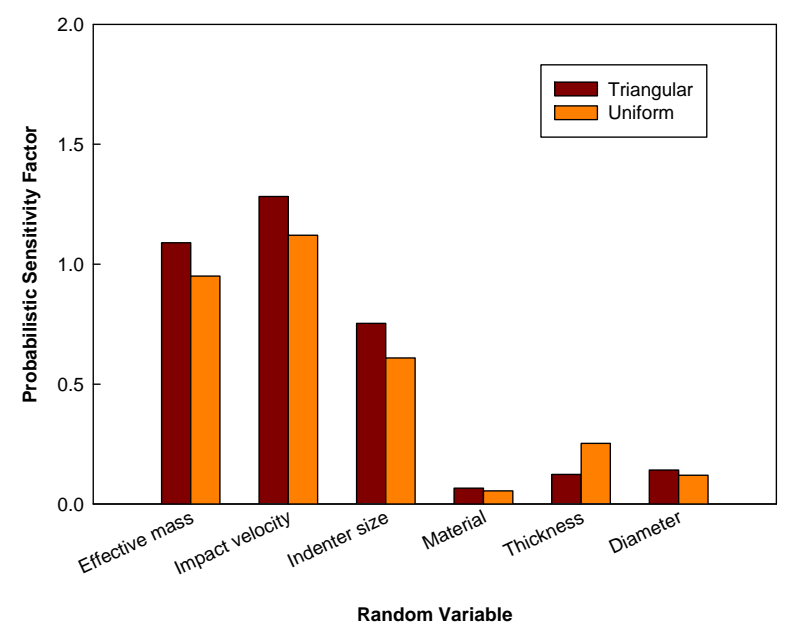

Figure 8. Probabilistic Sensitivity with Respect to Changes in Standard Deviation
Moreover, the relative ranking of the random variables is consistent for each measure of probabilistic sensitivity. However, the ranking order between tank thickness and tank diameter varies depending on the assumed distribution function for thickness (Table 4). Regardless of the assumed distribution for thickness, the probabilistic sensitivity analysis indicates that the three most significant factors affecting conditional probability of release are impact velocity, effective collision mass, and indenter size.

Table 4. Relative Ranking of Factors Affecting Conditional Probability of Release

Assumed Distribution for Tank Thickness

\begin{tabular}{lll}
\cline { 2 - 3 } & Triangular & Uniform \\
\hline (1) & Impact velocity & Impact velocity \\
(2) & Effective mass & Effective mass \\
(3) & Indenter size & Indenter size \\
(4) & Tank diameter & Tank thickness \\
(5) & Tank thickness & Tank diameter \\
(6) & Material & Material
\end{tabular}

\section{DISCUSSION}

In this paper, results from the probabilistic model are calibrated to produce trends that are consistent with those observed in accident data as tank head thickness is varied. That is, conditional probability of release from head impacts tends to decrease as head thickness increases. The calibration procedure entails inverse or reverse engineering to backcalculate mean values and standard deviations for the random variables assumed in the model as well as constants for puncture energy. Model results are within the 95 percent confidence bounds of the logistic regression results from the available accident data for tank heads without head shields and without jackets (Figure 3). The effect of jackets and head shields is incorporated into the model by adding jacket/shield thickness to head thickness. The discrepancy between model results and accident data for jacketed tank heads without head shields (Figure 4) suggests that other factors may contribute to the impact mechanics which have not been addressed in the probabilistic model. This hypothesis, however, is not supported in the case of unjacketed tank heads with head shields where the results from the model without accounting for other factors are within excellent agreement with the accident data (Figure 5).

Probabilistic sensitivity analysis indicates that the most significant factors affecting conditional probability of release are impact velocity, effective collision mass, and indenter size. The relative ranking of these factors is based upon the various assumptions made in developing the model, but appears reasonable. For example, the strong effect of speed is consistent with results from a previous study on the quantitative estimation of risk associated with the transportation of hazardous materials (hazmat) by rail [8]. In the previous study, speed is shown to have a three-fold effect 
on hazmat accidents. As speed increases: (1) the number of derailing cars increases, thus increasing the probability that hazmat cars present in the train will derail, (2) the probability that a derailed hazmat car will release its contents increases, and (3) the amount of hazmat from a releasing car increases. The strong effect of indenter size is consistent with results from deterministic sensitivity studies of puncture resistance to tank car shell impacts based on finite element analysis [9]. In addition, deterministic sensitivity analysis of pipeline puncture during excavations indicates that puncture is strongly dependent on tooth size (i.e., indenter size), pipe wall thickness, and material grade $[10,11]$.

Two of the three factors having the most significant effect of conditional probability of release; namely, effective collision mass and indenter size; are often unknown in actual accidents. For example, in composite summaries of major railroad accidents involving the release of hazardous materials between 1969 and 1978 [12], the probable impacting object was reported in 22 of 75 accidents. In these 22 accidents, 13 were couplers, five were drawbars, two were broken rails, one was a wheel, and one was an end sill of a car.

The relatively weak effect of thickness, compared to other factors, is examined by assuming different probability functions, specifically triangular and uniform. The ranking order between tank thickness and tank diameter is altered depending on the assumed distribution, but the order of the three most significant factors affecting conditional probability of release is unchanged.

The use of conditional probability of release as a performance metric stems from its direct applicability to perform risk assessments and cost/benefit analysis. However, its usefulness may become problematic if and when enhanced designs that apply technologies beyond those used in currently accepted practice (e.g., impact energy absorbing structures) become practical alternatives to conventional designs. Estimates for the conditional probability of release of hazardous materials from tank car with alternative designs must be based on extrapolations and simplifying assumptions initially due to the lack of a sufficient database of accident and failure experience.

Conditional probability of release considers the probability of lading loss but does not take into account the amount of lost commodity during the accidental release of hazardous material. Moreover, no attempt has been made in this paper to examine the quantity of lading loss in accidents.

\section{CONCLUDING REMARKS}

This paper describes a probabilistic approach to estimate conditional probability of release of hazardous materials from tank car head impacts during accidents. Monte Carlo methods are used in the model to reproduce trends that mimic those observed in available accident data compiled over a forty-year period. A probabilistic sensitivity analysis is performed with the calibrated model to examine the relative effect of the various factors assumed in the model on conditional probability of release. Sensitivity is calculated on the basis of three different measures. The relative ranking of the most significant factors affecting conditional probability of release is consistent for each measure of probabilistic sensitivity. Moreover, results from the probabilistic sensitivity analysis suggest that the three most significant factors that affect conditional probability of release are impact velocity, effective collision mass, and indenter size.

\section{ACKNOWLEDGMENTS}

The research described in this paper was sponsored by the Federal Railroad Administration, Office of Research and Development under the Rail Equipment Safety Research Program. Mr. Francisco Gonzalez III of the Equipment and Operating Practices Division is the Program Manager for research related to railroad tank car safety.

\section{REFERENCES}

[1] Treichel, T., Hughes, J.P., Barkan, C.P.L., Sims, R.D., Phillips, E.A., Saat, M.R., Wen, Y.K., Simpson, D.G., "Safety Performance of Tank Cars in Accidents: Probability of Lading Loss,” RSI-AAR Railroad Tank Car Safety Research and Test Project Report No. RA 0502, January 2006.

[2] Hughes, J.P., Heuer, C.F., Anderson, T.L., "Fracture Behavior of Tank Car Steels in Accidents from 1981 through 1994," RSI-AAR Railroad Tank Car Safety Research and Test Project Report No. RA 03-6-62, December 1998.

[3] Anderson, T.L., "Probabilistic Fracture Mechanics Analysis of Lading Loss in Railroad Tank Cars," $43^{\text {rd }}$ Mechanical Working and Steel Processing Conference Proceedings, Iron and Steel Society, Vol. XXXIX, October 2001, pp. 883-894.

[4] Sims, R.D., Hughes, J.P., "Steels for Tank Cars," $43^{\text {rd }}$ Mechanical Working and Steel Processing Conference Proceedings, Iron and Steel Society, Vol. XXXIX, October 2001, pp. 825-835.

[5] Haldar, A., Mahadevan, S., Probability, Reliability and Statistical Methods in Engineering Design, John Wiley \& Sons, 2000.

[6] Palmer, A., Neilson, A., Sivadasan, S., "Impact resistance of pipelines and the loss-of-containment limit state,” Journal of Pipeline Integrity 2, 2003, pp. 231240.

[7] McKeighan, P.C., "Mechanical Properties of Tank Car Steels Retired from the Fleet," Southwest Research Institute Report to Volpe Center, August 2008.

[8] Nayak, P.R., Rosenfield, D.B., Hagopian, J.H., “Event Probabilities and Impact Zones for Hazardous Materials Accidents on Railroads," Final Report No. DOT/FRA/ORD-83/20, November 1983.

[9] Tang, Y.H., Yu, H., Gordon, J.E., Jeong, D.Y., “Analysis of Railroad Tank Car Shell Impacts Using Finite Element 
Method," Proceedings of the 2008 IEEE/ASME Joint Rail Conference, JRC2008-63014, April 2008.

[10] Brooker, D.C., "Numerical modeling of pipeline puncture under excavator loading, Part I: development and validation of finite element material failure model for puncture simulation," International Journal of Pressure Vessels and Piping 80, 2003, pp. 715-725.

[11] Brooker, D.C., "Numerical modeling of pipeline puncture under excavator loading, Part II: parametric study," International Journal of Pressure Vessels and Piping 80, 2003, pp. 727-735.

[12] Glickman, T.S., "Major Railroad Accidents Involving Hazardous Materials Release, Composite Summaries, 1969-1978,” Report No. FRA-RRS-80-04, July 1980.

[13] Ayyub, B.M., McCuen, R.H., Probability, Statistics \& Reliability for Engineers, CRC Press, 1997.

[14] Wu, Y.T., "Computational methods for efficient structural reliability and reliability sensitivity analysis," AIAA Journal 32, August 1994, pp. 1717-1723.

[15] Mohanty, S., Wu, Y.T., "CDF sensitivity analysis technique for ranking influential parameters in the performance assessment of the proposed high-level waste repository at Yucca Mountain, Nevada, USA,” Reliability Engineering and System Safety 73, 2001, pp. 167-176.

\section{APPENDIX A - PROBABILITY FUNCTIONS}

This appendix lists the probability density functions (PDF) and cumulative distribution functions (CDF) for the different distributions considered in the probabilistic model described in this paper.

\section{Normal Distribution}

The PDF for a normal or Gaussian distribution is

$$
f(x)=\frac{1}{\sigma \sqrt{2 \pi}} \exp \left[-\frac{(x-\mu)^{2}}{2 \sigma^{2}}\right]
$$

where $\mu$ is the mean value and $\sigma$ is the standard deviation. The corresponding CDF is

$$
F(x)=\int_{-\infty}^{x} \frac{1}{\sigma \sqrt{2 \pi}} \cdot \exp \left[-\frac{(x-\mu)^{2}}{2 \sigma^{2}}\right] d x=\Phi\left[\frac{(x-\mu)}{\sigma}\right]
$$

where $\Phi$ is the standard error function which is defined as

$$
\Phi(z)=\frac{1}{\sqrt{2 \pi}} \int_{-\infty}^{z} \exp \left(-\frac{1}{2} \varsigma^{2}\right) d \varsigma
$$

$$
f(x)=\frac{1}{\varsigma x \sqrt{2 \pi}} \cdot \exp \left[-\frac{(\ln (x)-\lambda)^{2}}{2 \varsigma^{2}}\right]
$$

where $\lambda$ and $\zeta$ are parameters that are related to the mean $\mu$ and standard deviation $\sigma$ through the following equations:

$$
\begin{aligned}
& \mu=\exp \left(\lambda+\frac{1}{2} \varsigma^{2}\right) \\
& \sigma=\sqrt{\exp \left(2 \lambda+\varsigma^{2}\right)\left[\exp \left(\varsigma^{2}\right)-1\right]}
\end{aligned}
$$

The corresponding CDF for a log normal distribution can be expressed in terms of the standard normal integral as

$$
F(x)=\Phi\left[\frac{\ln (x)-\lambda}{\varsigma}\right]
$$

\section{Weibull Distribution}

The three-parameter Weibull probability distribution is defined as

$$
f(x)=\frac{\beta}{(\eta-\delta)}\left(\frac{x-\delta}{\eta-\delta}\right)^{\beta-1} \exp \left[-\left(\frac{x-\delta}{\eta-\delta}\right)^{\beta}\right]
$$

where $\beta$ is called the shape parameter, $\eta$ is called the scale parameter, and $\delta$ is called the location parameter. For a twoparameter Weibull distribution, the location parameter $\delta$ is equal to zero, and the Weibull shape and scale parameters are related to the mean value $\mu$ and the standard deviation $\sigma$ through the following relations:

$$
\begin{aligned}
& \mu=\eta \cdot \Gamma\left(1+\frac{1}{\beta}\right) \\
& \sigma=\eta \cdot \sqrt{\Gamma\left(1+\frac{2}{\beta}\right)-\left[\Gamma\left(1+\frac{1}{\beta}\right)\right]^{2}}
\end{aligned}
$$

where $\Gamma$ is the complete gamma function which is defined as

$$
\Gamma(x)=\int_{0}^{\infty} e^{-\zeta} \zeta^{x-1} d \zeta
$$

The CDF for the three-parameter Weibull distribution is

\section{Log Normal Distribution}

The PDF for a log normal distribution is 
$F(x)=1-\exp \left[-\left(\frac{x-\delta}{\eta-\delta}\right)^{\beta}\right]$

\section{Triangular Distribution}

The PDF for a triangular distribution is

$$
f(x)=\left\{\begin{array}{l}
\frac{2\left(x-x_{\min }\right)}{\left(x_{\text {mode }}-x_{\text {min }}\right)\left(x_{\text {max }}-x_{\text {min }}\right)} \text { if } x_{\text {min }} \leq x \leq x_{\text {mode }} \\
\frac{2\left(x_{\text {max }}-x\right)}{\left(x_{\text {max }}-x_{\text {mode }}\right)\left(x_{\text {max }}-x_{\text {min }}\right)} \text { if } x_{\text {mode }}<x \leq x_{\text {max }}
\end{array}\right.
$$

where $x$ must lie between $x_{\min }$ and $x_{\max }$, and $x_{\text {mode }}$ is the most likely value. For the triangular probability distribution, the mean and standard deviation are related to the minimum, maximum, and most likely values through the following equations:

$\mu=\frac{1}{3}\left(x_{\min }+x_{\text {mode }}+x_{\max }\right)$

$\sigma=\sqrt{\frac{1}{18}\left(x_{\min }^{2}+x_{\text {mode }}^{2}+x_{\max }^{2}-x_{\min } x_{\text {mode }}-x_{\text {min }} x_{\text {max }}-x_{\text {mode }} x_{\text {max }}\right)}$

The corresponding CDF for a triangular distribution is

$$
F(x)= \begin{cases}0 & \text { if } x \leq x_{\text {min }} \\ \frac{\left(x-x_{\text {min }}\right)^{2}}{\left(x_{\text {mode }}-x_{\text {min }}\right)\left(x_{\text {max }}-x_{\text {min }}\right)} & \text { if } x_{\text {min }}<x \leq x_{\text {mode }} \\ 1-\frac{\left(x_{\text {max }}-x\right)^{2}}{\left(x_{\text {max }}-x_{\text {mode }}\right)\left(x_{\text {max }}-x_{\min }\right)} & \text { if } x_{\text {mode }}<x \leq x_{\text {max }} \\ 1 & \text { if } x>x_{\text {max }}\end{cases}
$$

\section{Uniform Distribution}

The PDF for a uniform distribution is

$f(x)= \begin{cases}\frac{1}{16\left(x_{\text {max }}-x_{\text {min }}\right)} & \text { if } x_{\text {min }} \leq x \leq x_{\text {max }} \\ 0 & \text { otherwise }\end{cases}$

The mean value and standard deviation are calculated from the minimum and maximum values:

$$
\mu=\frac{1}{2}\left(x_{\min }+x_{\max }\right)
$$

$$
\sigma=\frac{1}{12}\left(x_{\max }-x_{\min }\right)^{2}
$$

The corresponding CDF is

$$
F(x)= \begin{cases}0 & \text { if } x \leq x_{\min } \\ \frac{x-x_{\text {min }}}{x_{\max }-x_{\text {min }}} & \text { if } x_{\min }<x \leq x_{\text {max }} \\ 1 & \text { if } x>x_{\text {max }}\end{cases}
$$

\section{APPENDIX B - PROBABILISTIC SENSITIVITY}

This appendix summarizes the concepts and describes the mathematical calculations that were carried out to perform the probabilistic sensitivity analysis in this paper.

In probabilistic structural analysis, the measure of risk is commonly expressed in terms of the probability of failure. The probability of failure of a structure is determined from a specific performance criterion that depends on the relevant loading and structural resistance (i.e. strength) parameters that are treated as random variables. A response function is generally defined as a function of a vector of random variables

$$
Z(\underline{X})=Z\left(X_{1}, X_{2}, \ldots X_{n}\right)
$$

where the $X_{i}$ 's are the random variables and $\underline{X}$ represents the vector of random variables. The corresponding performance function defines the failure surface or limit-state that characterizes the assumed failure criterion

$g=Z(\underline{X})-Z_{0}=0$.

The performance function or limit-state function is written such that values of $g$ less than zero represent failure. Therefore, the probability of failure is related to the performance function by

$$
p=P[g<0]=\int \cdots \int_{g(\underline{x}) \leq 0} f_{x}(\underline{x}) d \underline{x}
$$

where $f_{X}$ is the joint probability density function.

In this paper, the multiple integral in equation (A-23) is evaluated through Monte Carlo procedures. Moreover, the performance function assumed is defined as

$$
g=E_{p}-E_{K}
$$


where $E_{p}$ is the puncture resistance in terms of energy and $E_{K}$ is the applied accident loading in terms of kinetic energy. The factors affecting energy to puncture or the resistance against puncture in terms of energy and their functional relation are expressed through the following equation

$E_{P}=C P^{a} \sigma_{U} h^{b} D^{c}$

where $P$ is the perimeter of the indenter, $\sigma_{U}$ is the ultimate tensile strength of the tank car steel, $h$ is the tank thickness, and $D$ is the tank diameter. In addition, $C, a, b$, and $c$ are constants. The functional form of this equation for puncture resistance is similar to that used in semi-empirical analysis of puncture during excavation of pipelines [6].

The factors affecting kinetic energy and their functional relation are given by

$$
E_{K}=\frac{1}{2} m_{e} v_{i}^{2}
$$

where $m_{e}$ is the effective collision mass and $v_{i}$ is the impact velocity.

In general, the following mathematical problem must be solved:

Minimize $\beta=\sqrt{\sum_{i=1}^{n}\left(x_{i}^{\prime}\right)^{2}}$ such that $g\left(\underline{x}^{\prime}\right)=0$

where $\underline{x}^{\prime}$ refers to the vector of reduced random variables, which is defined below. Moreover, equation (A-27) is usually solved using nonlinear optimization methods. Alternatively, the solution can be achieved iteratively by solving the following set of simultaneous equations in an iterative procedure:

$\alpha_{i}=\left(\frac{\partial g}{\partial X_{i}^{\prime}}\right)_{*} / \sqrt{\sum_{i=1}^{n}\left(\frac{\partial g}{\partial X_{i}^{\prime}}\right)_{*}^{2}}$

$x_{i}^{\prime *}=-\alpha_{i}^{*} \beta$

$g\left(x_{1}^{\prime *}, x_{2}^{\prime *} \ldots x_{n}^{\prime *}\right)=0$.

The subscript or superscript of * means that quantity or expression is evaluated at the design point. Mathematically, equation (A-28) represents the directional cosines of the tangent hyperplane in the reduced coordinates. The directional cosines are indicators of the sensitivity of the performance function to variations in the random variables.

The following computational steps can be applied to solve equations (A-28) to (A-30), which is described by Ayyub and
McCuen [13] as an advanced first-order second-moment (AFOSM) method:

1. Assume an initial value for the design point. In the present analysis, the mean values of the random variables were chosen as the initial guess. The design point in the reduced coordinates is calculated using

$x_{i}^{\prime}=\frac{x_{i}-\mu_{x_{i}}}{\sigma_{x_{i}}}=u_{i}$.

2. Evaluate the directional cosines at the failure point. The partial derivatives to calculate the directional cosines are given by

$\left(\frac{\partial g}{\partial X_{i}^{\prime}}\right)=\left(\frac{\partial g}{\partial X_{i}}\right) \cdot\left(\frac{\partial X_{i}}{\partial X_{i}^{\prime}}\right)=\left(\frac{\partial g}{\partial X_{i}}\right) \sigma_{x_{i}}$

3. Solve the following equation for the root $\beta$ :

$g\left[\left(\mu_{x_{1}}-\alpha_{1} \sigma_{x_{1}} \beta\right),\left(\mu_{x_{2}}-\alpha_{2} \sigma_{x_{2}} \beta\right) \cdots\left(\mu_{x_{n}}-\alpha_{n} \sigma_{x_{n}} \beta\right)\right]=0$

4. Using the value of $\beta$ from step 3, evaluate a new design point using the following equation:

$x_{i}^{*}=\mu_{X_{i}}-\alpha_{i}^{*} \sigma_{X_{i}} \beta$

5. Repeat steps 1 to 4 until convergence of $\beta$ is obtained. The value of $\beta$ is referred to as the reliability index.

Sensitivity measures can be used to quantify the influence of each random variable on the probability of failure. One measure of sensitivity is called the probabilistic importance factor, which is equivalent to the normalized gradient vector of the performance function in the space of the standard normal variables

$\alpha=\frac{\nabla g}{|\nabla g|}$

This equation is equivalent to the vector for the direction cosines of the random variables; i.e., equation (A-28). The direction cosines are also related to the reliability index:

$\alpha_{i}=\frac{u_{i}^{*}}{\beta}=\left.\frac{\partial \beta}{\partial u_{i}}\right|_{X}$.

The vector for the probabilistic importance factors also has the property that 
$\alpha_{1}^{2}+\alpha_{2}^{2}+\cdots+\alpha_{n}^{2}=1$

where $n$ is the number of random variables. Moreover, the probabilistic importance factors, $\alpha_{i}$, are used as one of the sensitivity measures in this document to determine the relative ranking of the random variables in the Monte Carlo model for tank cars in accidents.

Two other probabilistic sensitivity measures have been developed by Wu $[14,15]$ that calculate the change in the probability of failure, $p$, with respect to changes in the mean value and the standard deviation of the random variables:

$S_{\mu_{i}}=\frac{\partial p / p}{\partial \mu_{i} / \sigma_{i}}$

$S_{\sigma_{i}}=\frac{\partial p / p}{\partial \sigma_{i} / \sigma_{i}}$

where $\mu_{i}$ and $\sigma_{i}$ are the mean and standard deviation, respectively, of the random variables. The use of the standard deviation as a scale factor in these sensitivity measures implies that the allowable range of the mean value is limited to a local region characterized by the uncertainty of the random variable. Moreover, these sensitivity measures are dimensionless and can take positive, negative, or zero values. When $S_{\sigma}$ is zero or relatively small, it implies that the corresponding random variable can be varied over a wide range without significantly changing the probability of failure. This, in turn, implies that $S_{\mu}$ will be relatively small. On the other hand, when $S_{\sigma}$ is relatively large, the corresponding $S_{\mu}$ will also tend to be large, which suggests that $S_{\sigma}$ and $S_{\mu}$ are strongly related and both can be used to identify key contributing random variables. For a single limit state, $\alpha_{i}$ and $S_{\mu i}$ may be approximately proportional to $\alpha_{i}$ if $u_{i}{ }^{*}$ are relatively large.

In order to compute $S_{\mu}$ and $S_{\sigma}$, equations (A-38) and (A-39) are expressed as follows. The probability of failure is related to the reliability index through the following equation

$p=1-\Phi(\beta)=\Phi(-\beta)$

where $\Phi$ is the standard normal cumulative probability function, which was defined in equation (A-3). Equation (A-40) is differentiated with respect to the mean value $\mu$ $\frac{\partial p}{\partial \mu_{i}}=\frac{\partial}{\partial \mu_{i}}[\Phi(-\beta)] \cdot \frac{\partial \beta}{\partial \mu_{i}}=-\phi(-\beta) \cdot \frac{\partial \beta}{\partial \mu_{i}}$

where $\phi$ is the standard normal probability density function. Then applying the chain rule of differentiation,

$\frac{\partial p}{\partial \mu_{i}}=-\phi(-\beta) \frac{\partial \beta}{\partial u_{i}} \frac{\partial u_{i}}{\partial \mu_{i}}=-\phi(-\beta) \alpha_{i} \frac{\partial u_{i}}{\partial \mu_{i}}$

where

$\frac{\partial \beta}{\partial \mu_{i}}=\frac{\partial \beta}{\partial u_{i}} \frac{\partial u_{i}}{\partial \mu_{i}}=\alpha_{i} \frac{\partial u_{i}}{\partial \mu_{i}}$

Similarly, the derivative of equation (A-40) with respect to the standard deviation $\sigma$ is

$\frac{\partial p}{\partial \sigma_{i}}=\frac{\partial}{\partial \sigma_{i}}[\Phi(-\beta)] \cdot \frac{\partial \beta}{\partial \sigma_{i}}=-\phi(-\beta) \cdot \frac{\partial \beta}{\partial \sigma_{i}}$

Applying the chain rule,

$\frac{\partial p}{\partial \sigma_{i}}=-\phi(-\beta) \frac{\partial \beta}{\partial u_{i}} \frac{\partial u_{i}}{\partial \sigma_{i}}=-\phi(-\beta) \alpha_{i} \frac{\partial u_{i}}{\partial \sigma_{i}}$

where

$\frac{\partial \beta}{\partial \sigma_{i}}=\frac{\partial \beta}{\partial u_{i}} \frac{\partial u_{i}}{\partial \sigma_{i}}=\alpha_{i} \frac{\partial u_{i}}{\partial \sigma_{i}}$

Therefore, combining equations gives

$$
\begin{aligned}
& S_{\mu_{i}}=\frac{\partial p}{\partial \mu_{i}}\left(\frac{\sigma_{i}}{p}\right)=\frac{-\phi(-\beta)}{\Phi(-\beta)} \alpha_{i} \frac{\partial u_{i}}{\partial \mu_{i}} \sigma_{i} \\
& S_{\sigma_{i}}=\frac{\partial p}{\partial \sigma_{i}}\left(\frac{\sigma_{i}}{p}\right)=\frac{-\phi(-\beta)}{\Phi(-\beta)} \alpha_{i} \frac{\partial u_{i}}{\partial \sigma_{i}} \sigma_{i}
\end{aligned}
$$

Classical perturbation methods are applied to evaluate the partial derivatives of $u$ with respect to the mean and standard deviation. 